\title{
Hepatitis C Treatment in Chronic Kidney Disease Patients: The Kidney Disease Improving Global Outcomes Perspective
}

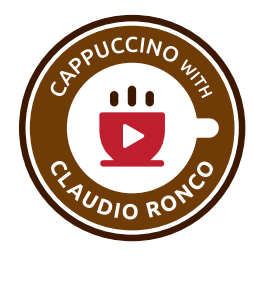

\author{
Michel Jadoul $^{\mathrm{a}} \quad$ Paul Martin $^{\mathrm{b}}$ \\ ${ }^{a}$ Division of Nephrology, Cliniques Universitaires Saint-Luc, Université Catholique de Louvain, Brussels, Belgium; \\ ${ }^{b}$ Division of Hepatology, University of Miami, Miami, FL, USA
}

\section{Key Words}

Hepatitis C virus - Hemodialysis - Chronic kidney disease .

Kidney transplantation · Direct-acting antiviral agent .

Sustained viral response

\begin{abstract}
Background: Hepatitis C virus (HCV) infection is a very common infection found among hemodialysis (HD) and kidney transplant patients. It is associated with substantial morbidity and mortality. Direct-acting antiviral agents (DAAs) have much better efficacy (sustained viral response (SVR)) and tolerance than interferon-based regimens. Very recent studies extend this breakthrough finding to chronic kidney disease (CKD) populations. Summary: CKD patients with an estimated glomerular filtration rate (eGFR) $>30 \mathrm{ml} / \mathrm{min} / 1.73 \mathrm{~m}^{2}$ can be treated with any licensed DAA regimen. In CKD stages 4-5 (mostly HD), the combination of grazoprevir (100 mg) and elbasvir $(50 \mathrm{mg})$, a once-daily oral regimen active against genotypes 1 and 4, induced in a very recent RCT an SVR rate $>95 \%$, with tolerance similar to that of placebo. Case series suggest that other DAA regimens are also very effective and well tolerated in HD patients. In kidney transplant recipients, 2 case series have reported 100\% SVR with good tolerance of sofosbuvir-based regimens. Importantly, there is a risk of drug-drug interaction of several DAAs including calcineurin inhibitors. Finally, the availability of $\mathrm{HCV}+$ grafts
\end{abstract}

\section{KARGER}

(c) 2017 S. Karger AG, Basel

E-Mail karger@karger.com

www.karger.com/bpu may markedly shorten the waiting time for transplantation. Key Messages: (1) In patients with an eGFR $>30$, all licensed DAAs regimens can be used. (2) Cure of HCV appears at hand in CKD stages 4-5, including dialysis patients, and in kidney transplant recipients. (3) The choice of DAA regimen in CKD should be based on HCV genotype, viral load, eGFR, concomitant medications, transplant candidacy and comorbidities. (4) The timing of treatment in potential kidney transplantation candidates (before versus after transplantation) should be decided in collaboration with the transplant center.

Video Journal Club 'Cappuccino with Claudio Ronco' at http://www.karger.com/?doi=452730. @ 2017 s. Karger AG, Basel

Hepatitis $\mathrm{C}$ virus (HCV) infection is much more prevalent in patients on renal replacement therapy than in the general population $[1,2]$. Several reasons concur to favor the treatment of HCV infection, both in patients on dialysis and in kidney transplant recipients. Indeed, $\mathrm{HCV}$ infection is associated with an increased risk of mortality in these patients $[3,4]$. In addition, HCV-induced de novo membrano-proliferative glomerulonephritis may impair allograft survival [5]. Finally, HCV antibody positivity, corresponding in most cases with the presence of active $\mathrm{HCV}$ infection, increases the incidence 
of hepatocellular carcinoma in kidney transplant recipients [6].

Major progress has been made over the last 2 decades with regard to the treatment of HCV infection among the general population. Sustained viral response (SVR) rates (which indicate cure of HCV infection) increased progressively from 7 to $10 \%$ with interferon alone, increased to $25 \%$ with interferon and ribavirin, and increased to $40-50 \%$ with peginterferon and ribavirin. SVR rates rose further to $60-70 \%$ with the addition of protease inhibitors and eventually to $>95 \%$ with the current direct-acting antiviral agents (DAAs). Importantly, DAA regimens are for shorter durations (mostly 12 weeks), are interferonfree and are thus found to have much improved tolerance [7].

Among the currently approved DAAs, sofosbuvir is the only one that has significant renal elimination. The other currently approved DAAs - simeprevir, ledipasvir, daclatasvir, paritaprevir/ritonavir, ombitasvir, dasabuvir, grazoprevir and elbasvir - are not eliminated by the kidneys and thus do not need dose adjustment, even in severe CKD or in hemodialysis (HD) patients $[8,9]$.

Thus, CKD patients with an estimated glomerular filtration rate $(\mathrm{eGFR})>30 \mathrm{ml} / \mathrm{min} / 1.73 \mathrm{~m}^{2}$, can be treated with any licensed DAA regimen [9], especially as a sizeable number of patients with CKD up to stage 3 were included in registration trials.

The choice of the regimen should be based on genotype (and subtype), viral load, concomitant medications, kidney function, transplant candidacy, and comorbidities.

In CKD stages 4-5, and especially in dialysis patients, progress in HCV treatment has been much slower. Indeed, until a few years ago, SVR rates with the (peg) interferon and ribavirin combinations were around $40 \%$, and tolerance was poor, prompting withdrawal of antiHCV treatment in close to $1 / 3$ of patients [10]. Despite these limitations, this early experience demonstrated the durability of virologic response even with a subsequent renal transplantation $[2,9]$. The limited efficacy and poor tolerance of interferon-based regimens probably largely account for the very low treatment rates of HCV in HD patients worldwide. Goodkin et al. [11] indeed used data of the Dialysis Outcomes and Practice Patterns Study (phases 1-4, corresponding to the (peg)-interferon (with or without ribavirin) era) to assess antiviral treatment rates across the 12 DOPPS countries (Canada, USA, 7 EU countries, Australia/NZ, Japan). Treatment rates were $<5 \%$, even in candidates for kidney transplantation.
Fortunately, evidence is now accumulating, showing that SVR rates may exceed $95 \%$ even in patients on renal replacement therapy.

Recently, Roth et al. [12] published the C-Surfer trial, a phase- 3 study that recruited 235 patients with CKD stage 4 or 5 (approximately $3 / 4$ on HD) with chronic HCV genotype 1 infection (the most prevalent genotype in dialysis patients). Only $6 \%$ of the patient population was cirrhotic and $80 \%$ were naïve to treatment. The study randomly assigned to either the immediate treatment group $(\mathrm{n}=111)$ or the delayed treatment group ( $\mathrm{n}=113)$ Grazoprevir (100 mg) and Elbasvir (50 mg), a once-daily oral combination for 12 weeks. In addition, a small group of patients $(\mathrm{n}=11)$ was assigned to the immediate treatment group but with intensive pharmacokinetic monitoring. Of note, a liver biopsy at baseline was not required as an inclusion criterion. Noninvasive biochemical markers or transient elastography were considered sufficient, in line with the evolution of clinical practice [13].

The design of this RCT contributed very informative results. All patients eventually benefited from the active treatment, but the inclusion of a control period (the delayed group) made it possible to assess in a randomized double-blind way, the tolerance of the study drug combination, as compared with the placebo period. Results are impressive both in terms of efficacy and tolerance. SVR 12 exceeded $95 \%$ in both the immediate and delayed treatment groups, and the study regimen was not withdrawn even in a single patient due to side effects [12].

At last, cure of HCV appears at hand in CKD stage 4 or 5 patients, including HD patients, at least those with genotype 1 or 4 infection (on which the grazoprevir/elbazvir regimen is active). Admittedly, like in most controlled trials, the study population was somewhat younger, and had lower comorbidities than the typical current dialysis population, but it appears reasonable to generalize these results to candidates for kidney transplantation. It should be mentioned that the trial did not include any patient on peritoneal dialysis. Thus, although pharmacokinetic characteristics of the study regimen do not suggest any specific problems in peritoneal dialysis as compared with HD, data are needed in this population. Recent case series further show that alternative drug regimens may be effective even in HD patients with HCV. Although the absence of the control group or period, unlike in the C-Surfer trial, complicates the precise assessment of the tolerance, these results are encouraging. Indeed, using the 3D regimen (that includes ombitasvirritonavir with paritaprevir and dasabuvir), Gomez et al. 
[14] recently reported in an abstract form an SVR rate of $100 \%$ in 21 patients, with acceptable tolerance, although drug-drug interactions with antihypertensive drugs were reported and anemia worsened in some patients, not requiring treatment discontinuation. In HCV genotype 1a infected patients, ribavirin may be needed with non-sofosbuvir-based regimens if naturally occurring resistance-associated variants are detected, in order to augment virological response rates. Importantly, however, sofosbuvir is not licensed for use in patients with a GFR $<30 \mathrm{ml} / \mathrm{min}$ so its use in advanced CKD is off label and more information about its toxicity in this setting is clearly needed before its use can be expanded in this population.

Two case series of Japanese HD patients (total $n=31$ ) with genotype 1 infection were very recently reported with the combination of daclatasvir and asunaprevir. SVR rates were $>95 \%$ in both series and tolerance was acceptable with a few SAE not ascribed by the investigators to the DAA regimen $[15,16]$. These results require confirmation in a larger series of patients.

The available evidence remains relatively scarce regarding how to best deal with other HCV genotypes (2, 3, 5 or 6). Some case series reported good results, with DAAs regimens active on these genotypes. These have included sofosbuvir, again raising concern about its use in advanced $C K D$.

A point of major interest to nephrologists is the significant risk of drug-drug interactions with several DAAs [8]. Briefly, cyclosporin-treated kidney HD patients were excluded from the C-Surfer trial [17] because of such a risk. Similarly, the 3D regimen impacts not only calcineurin inhibitors level, but also has a strong impact on the metabolism of drugs commonly prescribed to CKD patients, including dialysis and kidney transplant patients, such as amlodipine, statins and others [18].

In kidney transplanted patients, interferon-based regimens have long been known to be contraindicated. The choice of DAA regimen should be based on the genotype (and subtype), viral load, drug-drug interactions, CKD stage, stage of hepatic fibrosis, liver transplant candidacy, and comorbidities. The experience with the various DAA regimens remains limited as yet but is growing rapidly. It is very encouraging to note that 2 case series (total $n=45$; one from France, the other from the United States) reported $100 \%$ SVR rates with good tolerance, with various sofosbuvir-based DAA regimens. An impact on calcineurin inhibitors level (mostly tacrolimus) was reported in up to $40-50 \%$ of patients in both studies $[19,20]$.
Finally, a well-functioning kidney graft is associated with the best survival and quality of life in stage $5 \mathrm{CKD}$ patients. Thus, after the seminal Spanish experience of transplanting $\mathrm{HCV}+$ kidneys into $\mathrm{HCV}+$ recipients, there has recently been renewed interest in this strategy. Indeed in the United States [21], the waiting time for a deceased donor frequently exceeds 5 years, whereas patients may usually get an $\mathrm{HCV}+$ graft within a year. Of course, the applicability of this strategy will depend on the local epidemiology of HCV in deceased donors. Even more recently, some US transplant units have further started transplanting $\mathrm{HCV}+$ grafts into $\mathrm{HCV}$ - liver or kidney transplant recipients. This strategy, yet under investigation, is based on the close to $100 \%$ SVR rates with current DAA regimens, and the much shorter time to TP in those given an $\mathrm{HCV}+$ graft. Thus, anti-HCV treatment of renal transplant candidates should be undertaken in collaboration with the transplant center to optimize the timing of therapy.

In conclusion, the field of $\mathrm{HCV}$ treatment in $\mathrm{CKD}$ stages $4-5$, including dialysis and kidney transplant patients has changed drastically. HCV cure is now possible in most cases. This should help to reduce the prevalence of HCV in HD, and thus reduce further the risk of nosocomial transmission of HCV in HD units. Eradication $\mathrm{HCV}$ from HD units is now a realistic goal. These major changes should not deter the continuing efforts that are being put in to prevent the nosocomial transmission of $\mathrm{HCV}$, best reached with the cost-effective combination of prevention and treatment $[17,22]$.

\section{Disclosure Statement}

M. Jadoul has been a consultant for Merck. P. Martin has been a consultant and investigator for Gilead, Abbvie, Merck and Janssen.

References

1 Jadoul M: Transmission routes of HCV infection in dialysis. Nephrol Dial Transplant 1996;11(suppl 4):36-38.

2 Kidney Disease: Improving Global Outcomes (KDIGO): KDIGO clinical practice guidelines for the prevention, diagnosis, evaluation, and treatment of hepatitis $\mathrm{C}$ in chronic kidney disease. Kidney Int Suppl 2008; 109:S1-S99.

3 Fabrizi F, Takkouche B, Lunghi G, Dixit V, Messa P, Martin P: The impact of hepatitis C virus infection on survival in dialysis patients: meta-analysis of observational studies. J Viral Hepat 2007;14:697-703. 
4 Fabrizi F, Martin P, Dixit V, Bunnapradist S, Dulai G: Hepatitis $C$ virus antibody status and survival after renal transplantation: metaanalysis of observational studies. Am J Transplant 2005;5:1452-1461.

5 Cruzado JM, Carrera M, Torras J, Grinyó JM: Hepatitis $C$ virus infection and de novo glomerular lesions in renal allografts. Am J Transplant 2001;1:171-178.

6 Hoffmann CJ, Subramanian AK, Cameron AM, Engels EA: Incidence and risk factors for hepatocellular carcinoma after solid organ transplantation. Transplantation 2008; 86: 784-790.

7 Webster DP, Klenerman P, Dusheiko GM: Hepatitis C. Lancet 2015;385:1124-1135.

8 Kwo PY, Badshah MB: New hepatitis C virus therapies: drug classes and metabolism, drug interactions relevant in the transplant settings, drug options in decompensated cirrhosis, and drug options in end-stage renal disease. Curr Opin Organ Transplant 2015;20: 235-241.

9 Bhamidimarri KR, Martin P: Finally, safe and effective treatment options for hepatitis $\mathrm{C}$ in hemodialysis patients. J Hepatol 2016;65:7-10.

10 Gordon CE, Uhlig K, Lau J, Schmid $\mathrm{CH}$, Levey AS, Wong JB: Interferon treatment in hemodialysis patients with chronic hepatitis $C$ virus infection: a systematic review of the literature and meta-analysis of treatment efficacy and harms. Am J Kidney Dis 2008;51: 263-277.

11 Goodkin DA, Bieber B, Gillespie B, Robinson $B M$, Jadoul M: Hepatitis $C$ infection is very rarely treated among hemodialysis patients. Am J Nephrol 2013;38:405-412.

12 Roth D, Nelson DR, Bruchfeld A, Liapakis A, Silva M, Monsour H Jr, Martin P, Pol S, Londoño MC, Hassanein T, Zamor PJ, Zuckerman E, Wan S, Jackson B, Nguyen BY, Robertson M, Barr E, Wahl J, Greaves W: Grazoprevir plus elbasvir in treatment-naive and treatment-experienced patients with hepatitis $C$ virus genotype 1 infection and stage 4-5 chronic kidney disease (the C-SURFER study): a combination phase 3 study. Lancet 2015;386:1537-1545.

13 Jadoul M, Horsmans Y: Impact of liver fibrosis staging in hepatitis $\mathrm{C}$ virus (HCV) patients with kidney failure. Nephrol Dial Transplant 2014;29:1108-1110.

14 Gomez RM, Rincon D, Hernandez E, Ahumada A, Perez Valderas MD, Devesa MJ, Cuenca F, Fernandez C, Gutierrez ML, Ortiz M, Calvo M, Castellano G, Fernandez I: Ombitasvir/paritaprevir/ritonavir plus dasabuvir are safety and efficacy for treating HCV GT1 and 4 infection in patients with severe renal impairment or end-stage renal disease: a multicenter experience. J Hepatol 2016;64:S631S832, SAT-248.

15 Suda G, Kudo M, Nagasaka A, Furuya K, Yamamoto Y, Kobayashi T, Shinada K, Tateyama M, Konno J, Tsukuda Y, Yamasaki K, Kimura M, Umemura M, Izumi T, Tsunematsu S, Sato F, Terashita K, Nakai M, Horimoto H, Sho T, Natsuizaka M, Morikawa K, Ogawa K, Sakamoto N: Efficacy and safety of daclatasvir and asunaprevir combination therapy in chronic hemodialysis patients with chronic hepatitis C. J Gastroenterol 2016;51:733-740.

16 Miyazaki R, Miyagi K: Effect and safety of daclatasvir-asunaprevir combination therapy for chronic hepatitis $\mathrm{C}$ virus genotype $1 \mathrm{~b}$-infected patients on hemodialysis. Ther Apher Dial 2016;20:462-467.

17 Jadoul M, Horsmans Y: Towards eradication of hepatitis $\mathrm{C}$ virus from dialysis units. Lancet 2015;386:1514-1515.

18 Drug Interactions with Abbvie's 3D Regimen (Paritaprevir/Ritonavir, Ombitasvir and Dasabuvir). www.hcvdruginfo.ca (accessed September 19, 2016).

19 Kamar N, Marion O, Rostaing L, Cointault O, Ribes D, Lavayssière L, Esposito L, Del Bello A, Métivier S, Barange K, Izopet J, Alric L: Efficacy and safety of sofosbuvir-based antiviral therapy to treat hepatitis $C$ virus infection after kidney transplantation. Am J Transplant 2016;16:1474-1479.

20 Sawinski D, Kaur N, Ajeti A, Trofe-Clark J, Lim M, Bleicher M, Goral S, Forde KA, Bloom $\mathrm{RD}$ : Successful treatment of hepatitis $\mathrm{C}$ in renal transplant recipients with direct-acting antiviral agents. Am J Transplant 2016;16: 1588-1595.

21 Reese PP, Abt PL, Blumberg EA, Goldberg DS: Transplanting hepatitis C-positive kidneys. N ENgl J Med 2015;373:303-305.

22 Jadoul M, Fabrizi F: What are the management issues for hepatitis $\mathrm{C}$ in dialysis patients?: epidemiology and prevention of hepatitis $C$ virus transmission in the hemodialysis setting. Semin Dial 2014;27:449-451. 